\title{
Zero-dimensional superconducting fluctuations and fluctuating diamagnetism in lead nanoparticles
}

\author{
E. Bernardi, A. Lascialfari,* and A. Rigamonti \\ Department of Physics “A. Volta” and Unità CNISM-CNR, University of Pavia, Via Bassi 6, I-27100, Pavia, Italy \\ L. Romanò \\ Department of Physics and Unità CNISM-CNR, University of Parma, Parco Area delle Scienze 7A, I-43100, Parma, Italy \\ V. Iannotti and G. Ausanio \\ Coherentia CNR-INFM, Physical Sciences Department, “Federico II” University, Piazzale V. Tecchio 80, I-80125, Napoli, Italy \\ C. Luponio \\ Department of Materials Engineering and Production, "Federico II" University, Piazzale V. Tecchio 80, I-80125, Napoli, Italy
}

(Received 24 April 2006; revised manuscript received 3 August 2006; published 20 October 2006)

\begin{abstract}
High-resolution superconducting quantum interference device magnetization measurements in lead nanoparticles with particle size $d$ less than the superconducting coherence length $\xi$ are used to study zero-dimensional fluctuating diamagnetism. The diamagnetic magnetization $M_{\text {dia }}(H, T=$ const) as a function of the applied magnetic field $H$ at constant temperature is reported in the critical region and compared with the observed behavior in the temperature range where the first-order fluctuation corrections are expected to hold. The magnetization curves are analyzed in the framework of exact fluctuation theories based on the GinzburgLandau functional for $\xi \gg d$. The role of the upturn field $H_{\text {up }}$, where the slope of $M_{\text {dia }}(H)$ changes sign, is discussed. The relevance of the magnetization curves over a wide range of magnetic fields and the role of $H_{\text {up }}$ for the study of fluctuating diamagnetism, in particular, when the first-order fluctuation correction breaks down, is pointed out. The size and temperature dependences of $H_{\mathrm{up}}$ in the critical region are obtained from the experimental data and compared to the theoretical derivations for $M_{\text {dia }}$.
\end{abstract}

DOI: $10.1103 /$ PhysRevB.74.134509

\section{INTRODUCTION}

Superconducting fluctuations (SFs) and precursor effects occurring above the superconducting transition temperature $T_{c}$ have attracted great interest over the past few decades and resulted in advanced theoretical descriptions. ${ }^{1}$ The effect of an external magnetic field $H$ is especially interesting. While the field is a necessary tool to carry out relevant measurements, a strong field is expected to suppress the fluctuating Cooper pairs. Thus, a variety of phenomena are reflected in the dependence of the SF and of the fluctuating diamagnetism (FD) on the external field $H$. Moreover, experimentally studying the effect of $H$ on SF can be quite challenging, as for the cases of the field dependence of the spin susceptibility controlling the NMR spin-lattice relaxation ${ }^{2}$ or the diamagnetic susceptibility $\chi_{\text {dia }}$ related to $\mathrm{FD} .{ }^{3}$

A meaningful study of the field dependence of SF can be carried out in grains of size $d$ smaller than the coherence length $\xi$, i.e., in the zero-dimensional (OD) limit. In such samples one has an enhancement of the fluctuations relative to those at higher dimensions and an enlargement of the critical temperature regime. In 0D only spatially uniform fluctuations of the order parameter $\psi(\mathbf{r})$ need to be considered. Thus the Ginzburg-Landau (GL) free energy functional

$$
\begin{aligned}
F[\psi(\mathbf{r})]= & F_{\text {normal }}+\int d V\left\{a|\psi(\mathbf{r})|^{2}+(b / 2)|\psi(\mathbf{r})|^{4}\right. \\
& \left.+(1 / 4 m)|[-i(h / 2 \pi) \nabla-(2 e / c) \mathbf{A}] \psi(\mathbf{r})|^{2}\right\}
\end{aligned}
$$

where $^{1} a=\alpha_{0}\left[T-T_{\mathrm{c}}(0)\right]=\alpha_{0} T_{\mathrm{c}} \varepsilon, \mathbf{A}$ is the vector potential of the field, and $\varepsilon=\left[T-T_{\mathrm{c}}(0) / T_{\mathrm{c}}(0)\right]$ no longer requires a Fourier series expansion of $\psi(\mathbf{r})$ and so exact solutions for fields
PACS number(s): 74.40.+k, 74.20.De, 74.25.Ha

$H \ll H_{\text {c }}$ become possible. An understanding of the field dependence of SF in OD is important for the FD in bulk superconductors also, as the dominant contribution to the diamagnetic susceptibility $\chi_{\text {dia }}$ for $T \rightarrow T_{\mathrm{c}}^{+}$is due to metastable superconducting "droplets" of diameter of the order of $\xi(T)$. Then the isothermal magnetization curves $M_{\text {dia }}(T=$ const, $H$ ) can be qualitatively understood by assuming these fluctuating droplets are zero-dimensional. ${ }^{4,5}$

Attempts to study the magnetic aspects of the SF in metallic nanoparticles were carried out long ago, particularly through the electronic spin susceptibility $\chi_{\text {spin }}^{\prime \prime}(\mathbf{k}, \omega)$ via NMR $T_{1}$ relaxation measurements ${ }^{6,7}$ and measurements of the diamagnetic susceptibility $\chi_{\mathrm{dia}}{ }^{8}$ In $T_{1}$ measurements conclusive evidence for effects related to SF proved to be elusive, as the expected increase of the relaxation rate for $T$ $\rightarrow T_{\mathrm{c}}^{+}$was smeared out in 0D particles by a rounding of the transition and renormalization due to nonlinear fluctuations. ${ }^{7}$ For the Knight shift measurements it was difficult to isolate the contributions of SF, and again a reduction in $\chi_{\text {spin }}^{\prime \prime}(\mathbf{k}=0, \omega=0)$ in grains was observed near $T_{\mathrm{c}} \cdot{ }^{6,9}$

Fluctuation diamagnetism in aluminum nanoparticles was successfully studied in a pioneering experiment by Buhrman and Halperin ${ }^{8}$ by measuring the magnetization as a function of temperature, in constant magnetic fields. The data were analyzed in terms of $\chi_{\mathrm{dia}}$ for $H \rightarrow 0$, as derived in exact theories ${ }^{10,11}$ for the GL free energy functional in 0D limit. The behavior of $M_{\text {dia }}\left(T \approx T_{\mathrm{c}}\right)$ in nonzero magnetic fields was discussed by extending the zero-field equations with the replacement of the reduced temperature $\varepsilon$ by $\left(\varepsilon+H^{2} / H_{\mathrm{c}}^{2}\right)$, where $H_{\mathrm{c}}$ is the size-dependent critical field. The tendency toward the expected limiting slope $\chi_{\mathrm{dia}} \propto H^{-2}$ was emphasized. ${ }^{8}$ 
More recently, Li et al. ${ }^{12}$ have carried out conductivity, specific heat, and susceptibility measurements in $\mathrm{Pb}$ nanoparticles that have highlighted quantum size effects on superconducting (SC) properties. Electron tunnelling in nanometer-scale $\mathrm{Al}$ particles has been used ${ }^{13}$ to study the structure of the electronic energy levels. Size effects on $T_{\mathrm{c}}$ of lead nanoparticles embedded in an amorphous matrix have been studied by Tsai et al. ${ }^{14}$ Gladilin et al. ${ }^{15}$ have developed an exhaustive theory on the magnetic response and the SC properties of ultrasmall grains. For a description of the properties of ultrasmall particles, see the reviews in Ref. 16.

In this paper we report the results of a study of the superconducting fluctuations and of the related fluctuating diamagnetism in lead nanoparticles with diameters ranging from 150 to $750 \AA$, sizes for which effects of finite-level spacing should be negligible (see Sec. II). By means of high-field resolution superconducting quantum interference device (SQUID) measurements, isothermal magnetization curves $M_{\text {dia }}\left(H, T \approx T_{\mathrm{c}}^{+}\right)$were obtained and used to study the effect of magnetic field on the fluctuating pairs, particularly in the temperature range very near $T_{\mathrm{c}}$ where the first-order fluctuation correction breaks down and the role of the $|\psi(\mathbf{r})|^{4}$ term in Eq. (1) becomes crucial (the critical region). The importance of studying the magnetization curves on approaching $T_{\mathrm{c}}$ rather than the more common susceptibility measurements as a function of temperature at constant field has been noted for three-dimensional (3D) and two-dimensional (2D) superconductors. ${ }^{17}$ For metallic nanoparticles we will show that isothermal magnetization curves provide novel insights regarding critical fluctuations in the case of $0 \mathrm{D}$ as well.

\section{EXPERIMENTAL}

$\mathrm{The} \mathrm{Pb}$ nanoparticles were produced by a modification of the Polyol Process technique. ${ }^{18}$ All chemical reagents and solvents (Sigma-Aldrich products) had purity higher than 99\%. The reaction was performed in a sealed glass vessel surmounted by a cooling column in order to obtain polyol reflux. To produce a relatively narrow distribution of the nanoparticle dimensions, a high homogeneity of solution was obtained during the reaction process by vigorous mechanical stirring. $50 \mathrm{~mL}$ of tetraethylene glycol (TEG: $\mathrm{C}_{8} \mathrm{H}_{18} \mathrm{O}_{5}$ ) were introduced in the sealed vessel and heated by a thermostatic bath at $320^{\circ} \mathrm{C}$ to reach the TEG boiling. At this time a solution of $\mathrm{PbO}$ in TEG was introduced in the vessel. In order to produce grains of different sizes, the concentration of $\mathrm{PbO}$ in TEG and the reaction time were adjusted between 0.02 and $0.4 \mathrm{~mol}$ and $15-50 \mathrm{~min}$, respectively.

The powder was washed several times with ethanol at the beginning of the process and with acetone at the end. At the end of the reaction process the fine-powder fraction of $\mathrm{Pb}$ particles was separated from the coarse powder using a centrifuge. The particles were electrically isolated from one another by natural thin layers of oxide which were produced by aging the particles in atmosphere for about 30 days.

A variety of techniques was used to characterize the $\mathrm{Pb}$ powders. A Philips PW 1710 diffractometer utilizing $\mathrm{Cu} \mathrm{K} \alpha$ radiation $(\lambda=1.5418 \AA$ ) was used to carry out powder $\mathrm{x}$-ray diffraction (XRD) measurements. XRD patterns between $25^{\circ}<2 \theta<65^{\circ}$ were collected. A Philips EM 208S transmission electron microscope (TEM) with a copper $(\mathrm{Cu})$ grid coated with a formvar membrane was utilized at $100 \mathrm{keV}$ for imaging the nanoparticles. Lead $(\mathrm{Pb})$ nanoparticles were suspended in ethanol by sonicating, then the $\mathrm{Cu}$ grid was dipped in the solution. Finally, by means of an Edwards E306 metallizer, the grids containing the $\mathrm{Pb}$ nanoparticles were coated by a very thin carbon layer in order to avoid breaking the membrane. The nanoparticles were also analyzed through atomic force microscopy (AFM). The AFM (Digital Instruments Nanoscope IIIa) was equipped with a sharpened silicon tip with a radius less than $5 \mathrm{~nm}$. Images of the surface profiles were obtained by operating the AFM in the tapping mode, with a scan size and rate of $2 \mu \mathrm{m}$ and $2 \mathrm{~Hz}$, respectively.

The x-ray diffraction peaks in Fig. 1(a) are those expected for face-centered cubic (fcc) $\mathrm{Pb}$, with an additional smaller peak produced by the thin layer of superficial oxide, which is estimated to be approximately 5\% of the total grain weight. In the same figure (part $\mathrm{b}$ and part c) TEM images are shown. From these the oxide thickness can be estimated to be about $10 \%$ of the nanoparticle diameter. The particles size distribution, based on the TEM images and fitted to a lognormal distribution of the particle diameters, is shown in Fig. 1(d), for sample 1. In Fig. 2 the AFM image for sample 3 and the related particle size distribution, fitted to a log-normal distribution of the particle diameters, are given.

In Table I we report the expected diameter $d_{\mathrm{e}}=\exp (\mu$ $+\sigma^{2} / 2$ ), the standard deviation of the particle diameters $\sigma_{\mathrm{d}}$ $=\exp \left(\mu+\sigma^{2} / 2\right)\left[\exp \left(\sigma^{2}\right)-1\right]^{1 / 2}$, where $\mu$ and $\sigma$ are the mean and standard deviation of $\ln d$, respectively, treated as fitting variables, and the median diameter $d_{\mathrm{m}}$, obtained by finding the value at which the integral of the distribution given by a $\log$-normal function is equal on each side of $d_{\mathrm{m}}$.

The effects of finite-level spacing (see Ref. 16) are negligible for particle size $d \geqslant 100 \AA$ for the temperature range we are going to consider. The level spacing $\delta$ can be estimated from the inverse of the density of states at the Fermi level $\delta \approx 1 / N(0) v$, where $v$ is the nanoparticle volume. From $N(0)=(2 m)^{3 / 2} 8 \pi E_{\mathrm{F}}^{1 / 2} / h^{3}$ and for lead, with $E_{\mathrm{F}}=1.1$ $\times 10^{5}(K) k_{\mathrm{B}}$ and bulk value of the zero-temperature BCS gap $\Delta(0)=1.35 \mathrm{meV}$, one can write $\left(k_{\mathrm{B}} T_{\mathrm{c}} / \delta\right) \approx 3.4 \times 10^{-6} d^{3}$ (with $d$ in $\AA$ ). Thus, $\left(k_{\mathrm{B}} T_{\mathrm{c}} / \delta\right) \approx 1$ for $d \approx 70 \AA$, and only for particles of smaller size will the level spacing become comparable to $\Delta(0)$ and therefore invalidate the GL approach (see Refs. 16, 11, and 12).

From the thickness of the oxide layers, by assuming the condition of random loose packing the density of nanoparticles with respect to bulk $\mathrm{Pb}$ could be roughly estimated. Any uncertainty in the absolute value of the susceptibility per unit volume of lead does not affect the major conclusions to be derived in Sec. III.

The zero-field transition temperatures (see Table I) have been obtained by linearly extrapolating to zero the susceptibility in a field of 1 Oe plotted vs $T^{4}$ for $T \rightarrow T_{\mathrm{c}}^{-}$[inset in Fig. 3(a)]. Also in Fig. 3 a close-up of the data for $\chi_{\text {dia }}$, for a field of $2 \mathrm{Oe}$ and near $T_{\mathrm{c}}$, are shown as a function of the reduced temperature $\varepsilon$ for samples 1 and 3 .

The field dependence of the transition temperature $T_{\mathrm{c}}(H)$ has been estimated by extrapolating to zero the data for 


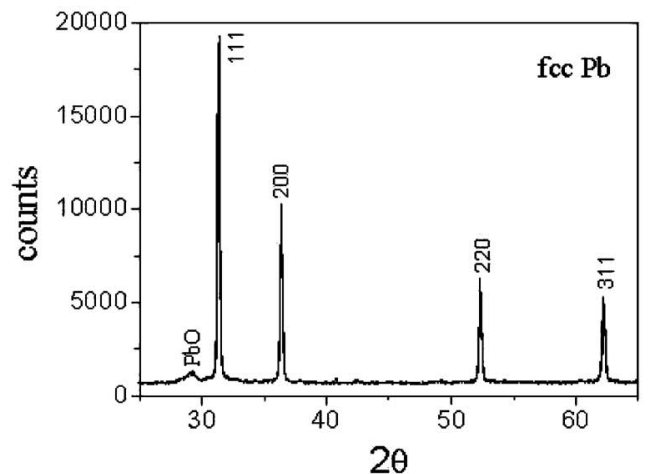

(a)

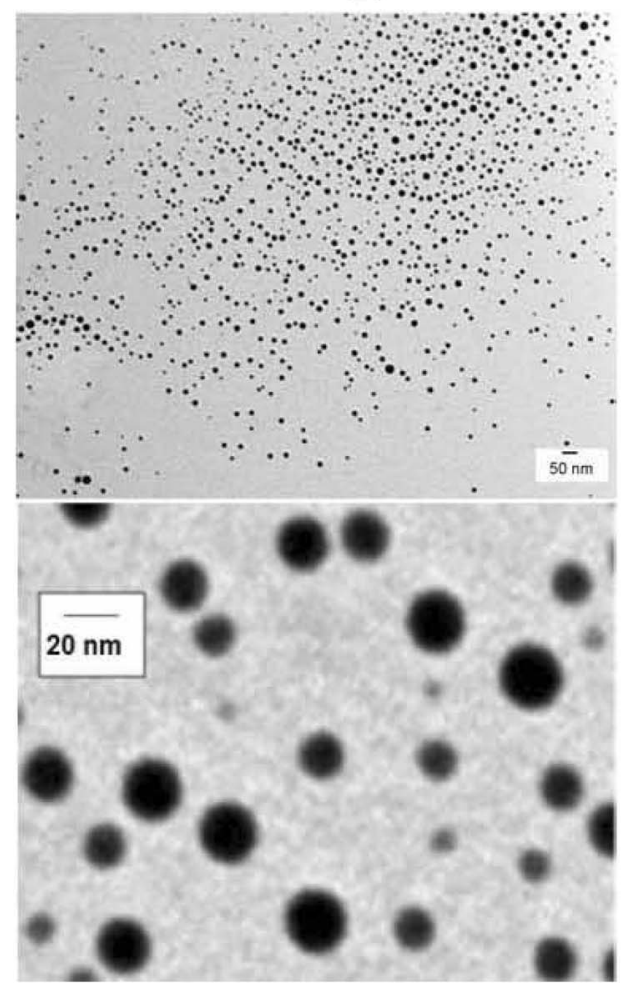

(b)

(c)

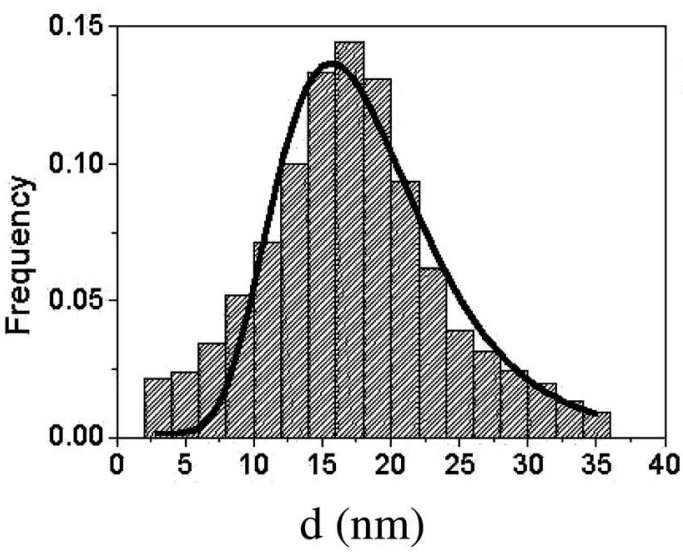

(d)

FIG. 1. (a) XRD of $\mathrm{Pb}$ nanopowder. (b) TEM images of $\mathrm{Pb}$ nanoparticles (mag. $40000 \times$ ). (c) TEM images of $\mathrm{Pb}$ nanoparticles (mag. $350000 \times$ ). (d) Size histogram of $\mathrm{Pb}$ nanoparticles produced under experimental conditions shown in (b). The curve in the part $d$ represents a log-normal distribution, which has a median diameter $d_{\mathrm{m} 1}=170 \AA$ (sample 1) and a standard deviation of the particle diameters $\sigma_{\mathrm{d} 1}=40 \AA$.

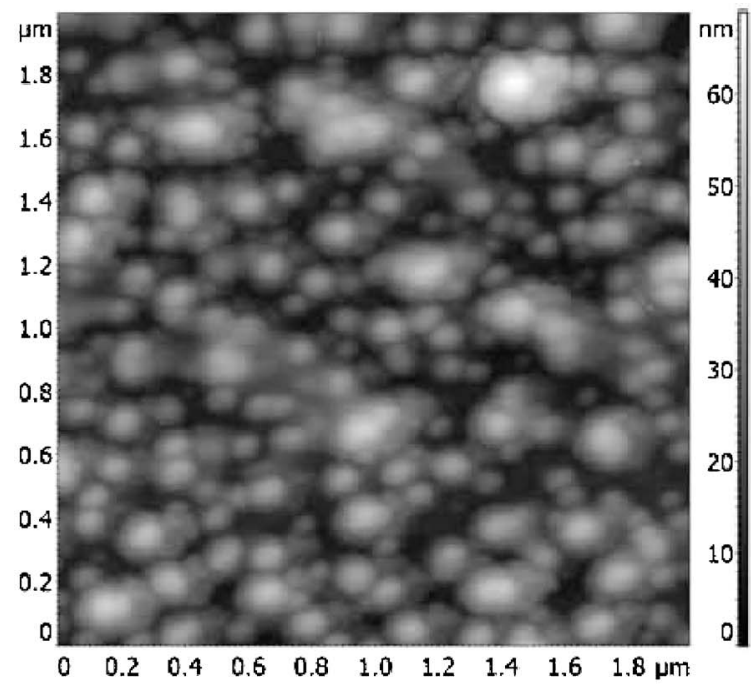

(a)

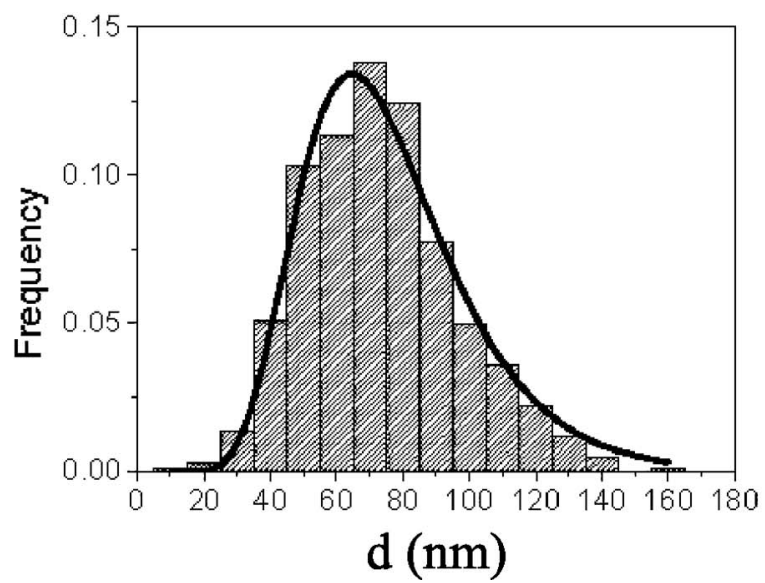

(b)

FIG. 2. (a) AFM image of $\mathrm{Pb}$ powder onto mica substrate obtained by increasing the concentration of $\mathrm{PbO}$ in TEG and the reaction time up to threefold. (b) Size histogram of $\mathrm{Pb}$ particles produced under experimental conditions shown in (a). The curve in the part (b) represents a log-normal distribution, which has a median diameter $d_{\mathrm{m} 3}=720 \AA$ (sample 3 ) and a standard deviation of the particle diameters $\sigma_{\mathrm{d} 3}=200 \AA$.

$\left(M_{\mathrm{dia}} / H\right)$ obtained in the temperature range where this quantity varies linearly with $T^{4}$ (Fig. 4).

In Fig. 5 representative isothermal magnetization curves are reported in the temperature range around $T_{\mathrm{c}}(0)$. The extraction of the diamagnetic contribution from the magnetization requires a detailed subtraction procedure when the magnetic field is increased to relatively strong values. In fact, in the range $H>H_{\text {up }},\left|M_{\text {dial }}\right|$ decreases on increasing the field (see Fig. 5), while the paramagnetic contributions due to the Pauli paramagnetism and to a small amount of paramagnetic impurities continue to increase on increasing $H$. Thus, from the computer-stored raw magnetization data around $T_{\mathrm{c}}(0)$ the magnetization values measured at a higher temperature (around $8 \mathrm{~K}$ ) where the $\mathrm{SF}$ are negligible have been subtracted. The slight variation of the paramagnetic contribution with temperature did not prevent reliable estimates of $M_{\text {dia }}$ for magnetic field up to about $600 \mathrm{Oe}$, as indicated by the error bars in Fig. 5(b). 
TABLE I. Expected (average) diameters of the particles, standard deviation of the particle diameters, median diameters of the particles, superconducting transition temperatures in the limit $H \rightarrow 0$ (in degrees $\mathrm{K}$ ), limits of the critical region (see text), and critical fields of the grains [as obtained from the extrapolation of $T_{\mathrm{c}}(H)$ for relatively low field according to Eq. (3)]. It is noted that by using an effective, properly averaged, diameter of the grains the estimates of $\varepsilon_{\mathrm{c}}$ do not change much.

\begin{tabular}{cccc}
\hline \hline$(1)$ & $(2 \mathrm{a})$ & $(2 \mathrm{~b})$ & $(3)$ \\
\hline$d_{\mathrm{e} 1}=160 \AA$ & $d_{\mathrm{e} 2}=250 \AA$ & $d_{\mathrm{e} 2}=400 \AA$ & $d_{\mathrm{e} 3}=750 \AA$ \\
$\sigma_{\mathrm{d} 1}=40 \AA$ & $\sigma_{\mathrm{d} 2}=60 \AA$ & - & $\sigma_{\mathrm{d} 3}=200 \AA$ \\
$d_{m_{1}}=170 \AA$ & - & - & $d_{m_{3}}=720 \AA$ \\
$T_{\mathrm{c}}=7.09 \pm 0.005$ & $T_{\mathrm{c}}=7.09 \pm 0.005$ & $T_{\mathrm{c}}=7.1 \pm 0.03$ & $T_{\mathrm{c}}=7.09 \pm 0.005$ \\
$\varepsilon_{\mathrm{c}} \approx 9 \times 10^{-2}$ & $\varepsilon_{\mathrm{c}} \approx 2.2 \times 10^{-2}$ & - & $\varepsilon_{\mathrm{c}} \approx 1.1 \times 10^{-2}$ \\
$H_{\mathrm{c}}^{\text {grain }} \approx 2500 \pm 400 \mathrm{Oe}$ & - & & $H_{\mathrm{c}}^{\text {grain }} \approx 1150 \pm 100 \mathrm{Oe}$ \\
\hline \hline
\end{tabular}

\section{ANALYSIS OF THE DATA}

The temperature range $\varepsilon<\varepsilon_{\mathrm{c}}$ where critical fluctuations are expected to occur and so the term in $|\psi(\mathbf{r})|^{4}$ in Eq. (1) becomes important, can be estimated according to slightly different criteria. The Ginzburg-Levanyuk criterion in 0D,
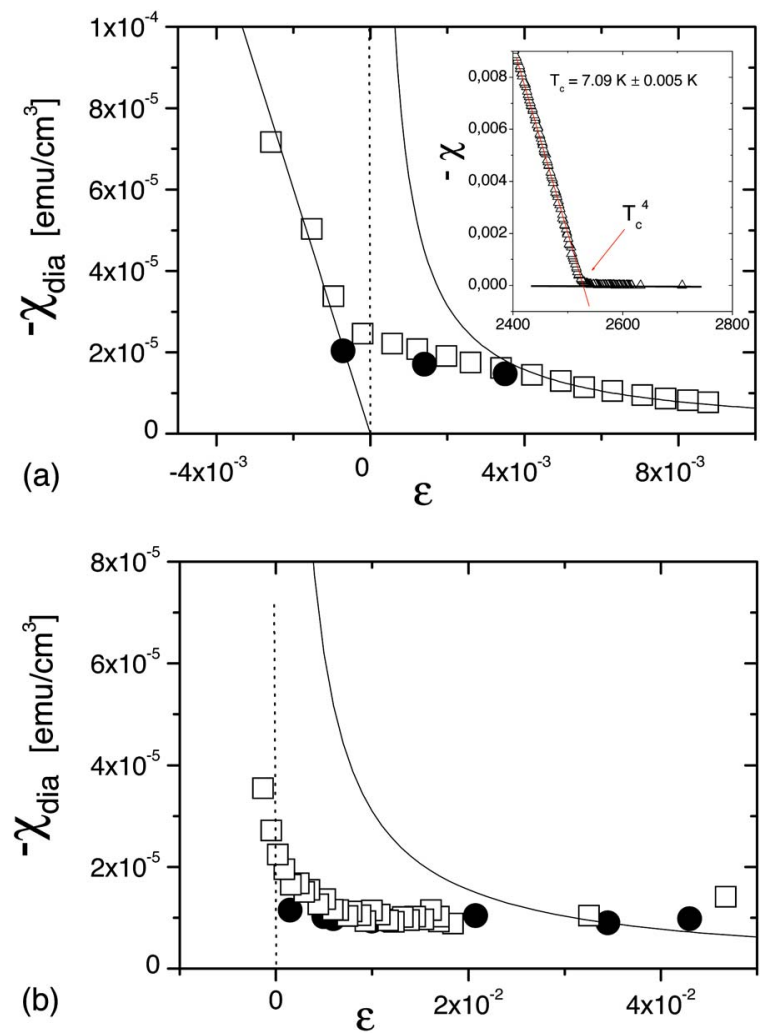

FIG. 3. (Color online) Blow-up of the temperature dependence of the susceptibility, in magnetic field $H=2 \mathrm{Oe}$, around $T_{\mathrm{c}}$ in sample 3 (a) and in sample 1 (b). The solid circles are the data from the isothermal magnetization curves while the empty squares are from the isofield measurements as a function of temperature. The solid lines track the behavior of $\chi_{\text {dia }}$ in the assumption that the nonlinear fluctuations can be neglected, namely, the behavior of $\left(M_{\mathrm{dia}} / H\right)$ for $H \rightarrow 0$, according to Eq. (2) in the text. The inset in Fig. 3(a) shows how the transition temperature has been determined. $\varepsilon$ is the reduced temperature. under the assumption of the BCS condition for $\xi_{0}$, yields ${ }^{1}$ $\varepsilon_{\mathrm{c}} \approx 13.3\left(T_{\mathrm{c}} / T_{\mathrm{F}}\right)\left(\xi_{0}^{3} / v\right)^{1 / 2}$, where $T_{\mathrm{F}}$ is the Fermi temperature, and $v$ the volume of the particle. Alternatively, the critical reduced temperature may be defined as the one below which the first-order fluctuation correction to the mean field behavior of $\left\langle|\psi|^{2}\right\rangle$ is no longer sufficient, ${ }^{11}$ in which case one obtains $\varepsilon_{\mathrm{c}} \approx 0.95\left[N(0) v k_{\mathrm{B}} T_{\mathrm{c}}\right]^{-1 / 2}$ where $N(0)$ is the singlespin density of states per unit volume. Assuming that the electron mean free path is limited by surface scattering, one then finds ${ }^{8} \varepsilon_{\mathrm{c}} \approx\left(6 k_{\mathrm{B}} T_{\mathrm{c}}\right)^{1 / 2} /(d / 2)^{3 / 2}\left|T_{\mathrm{c}} d H_{\mathrm{c}} / d T\right|_{\mathrm{Tc}}$. In lead the electron density is $n=1.32 \times 10^{23} \mathrm{~cm}^{-3}$ and $\xi_{0}=900 \AA$. The experimental result for the thermodynamic bulk critical field yields $\left|d H_{\mathrm{c}} / d T\right|_{\mathrm{Tc}}=81 \mathrm{Oe} / \mathrm{K}$. In Table I the average values of the critical temperature estimated according to the criteria described above are listed.

In the framework of the first-order fluctuation correction theory, which is valid for $\varepsilon>\varepsilon_{\mathrm{c}}$ and $H \ll H_{\mathrm{c}}$, the single particle magnetization is given by ${ }^{1}$

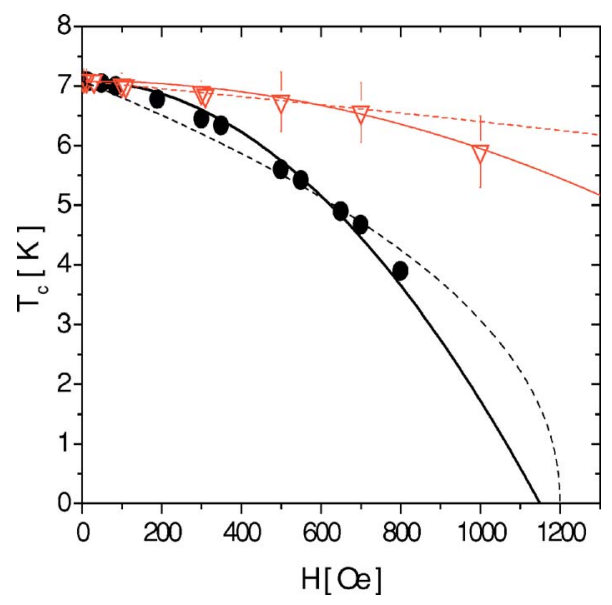

FIG. 4. (Color online) Field dependence of the superconducting transition temperatures for sample $3(\bullet)$ and for sample $1(\nabla)$. The dotted lines are the behaviors according to $H_{\mathrm{c}}(T)=H_{\mathrm{c}}(0)[1$ $\left.-\left(T / T_{\mathrm{c}}\right)^{\alpha}\right]$, with $\alpha=2.15$ and 2.2 , in correspondence to the critical field $1200 \mathrm{Oe}$ (for a sample at $d=860 \AA$ ) and for the indicative value $H_{\mathrm{c}}^{\text {grain }}(0)=5000$ Oe for $d=160 \AA$ (see Ref. 12). The solid line corresponds to Eq. (3) in the text and it appears to hold for $H$ $\leqslant H_{\mathrm{c}}^{\text {grain }}(0) / 2$. For sample 1 the extrapolation yields $H_{\mathrm{c}}^{\text {grain }}$ around 2500 Oe (see Table I). 


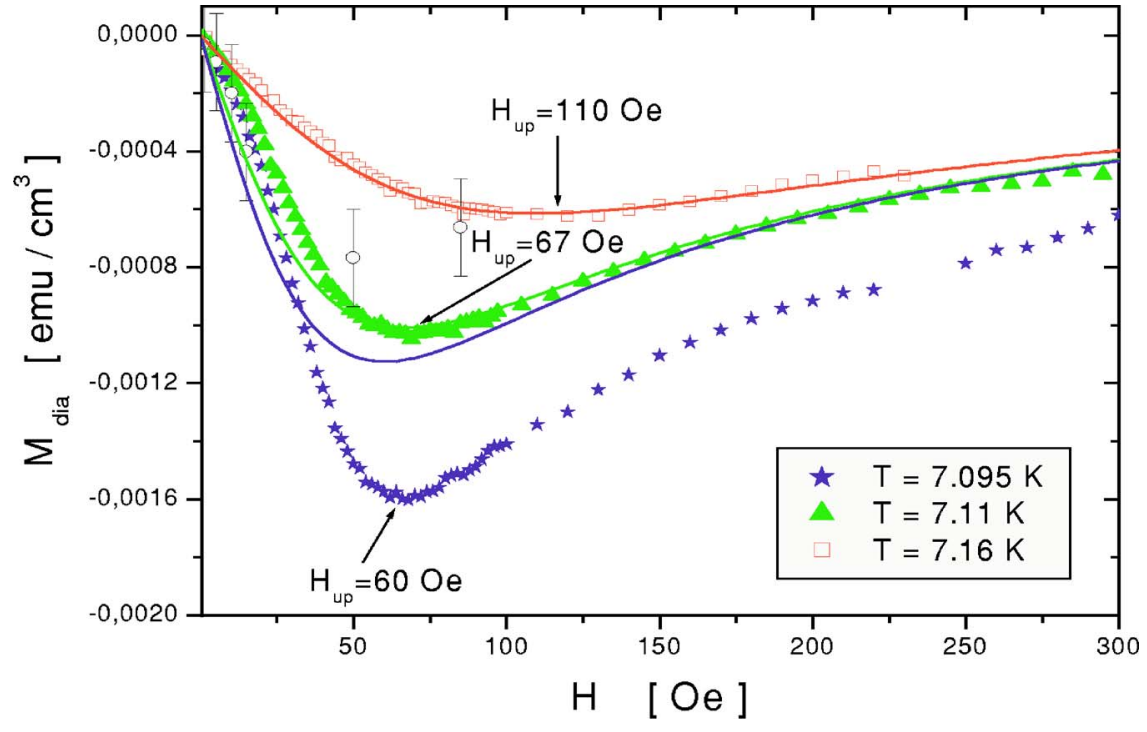

(a)

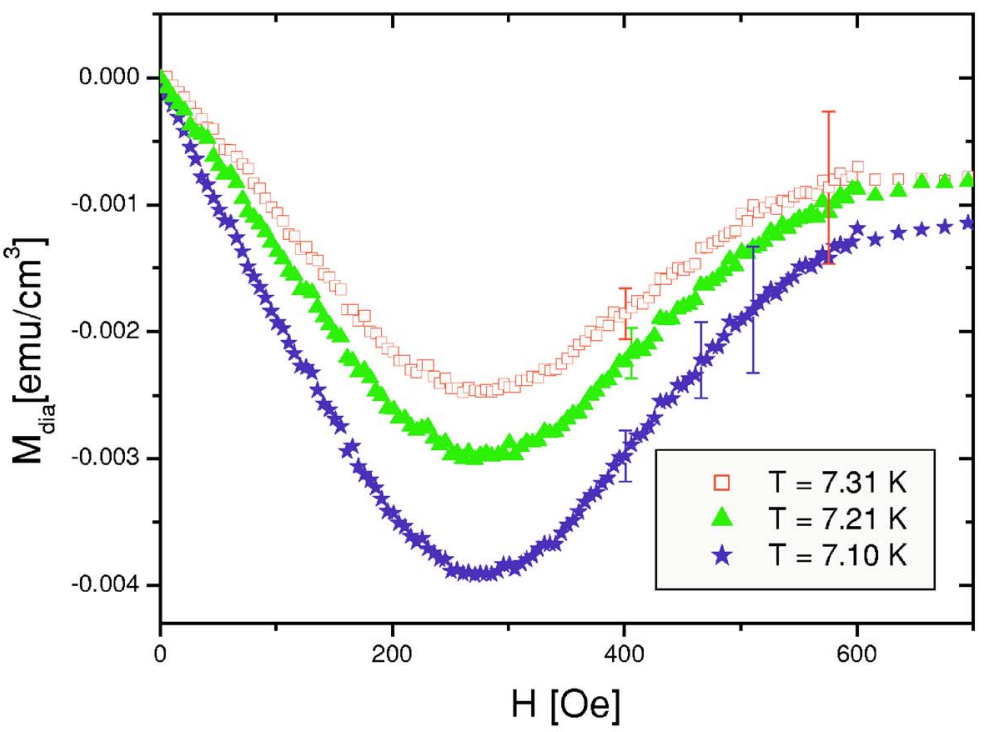

FIG. 5. (Color online) (a) Magnetization $M_{\text {dia }}$ vs $H$ in sample 3 at representative temperatures above $T_{\mathrm{c}}$ The solid lines correspond to Eq. (2) in the text for critical field of the grain 1150 Oe. Similar curves have been obtained for sample 2. For $\varepsilon \leqslant \varepsilon_{\mathrm{c}}$ the curves depart from the behavior described by Eq. (2). (b) Magnetization curves for sample 1 , all corresponding to temperature range where $\varepsilon<\varepsilon_{\mathrm{c}}$, namely, within the critical region. The open circles in part (a) correspond to the data obtained from the isofield measurements as a function of temperature, with large experimental errors.

(b)

$$
\begin{aligned}
M_{\text {dia }} & =-k_{B} T H\left(4 \pi^{2} \xi_{0}^{2} d^{2} / 5 \Phi_{0}^{2}\right) /\left[\varepsilon+\left(2 \pi^{2} \xi_{0}^{2} d^{2} H^{2} / 5 \Phi_{0}^{2}\right)\right] \\
& =-2 k_{B} T H\left(H_{\mathrm{c}}^{\text {grain }}\right)^{-2} /\left[\varepsilon+H^{2} /\left(H_{\mathrm{c}}^{\text {grain }}\right)^{2}\right] .
\end{aligned}
$$

$H_{\mathrm{c}}^{\text {grain }}=(2.5)^{1 / 2} \Phi_{0} / \pi \xi_{0} d$ is defined as the zero-temperature critical field of the grain. In fact, in the same theoretical scenario the field dependence of the superconducting transition temperature is given by

$$
\begin{aligned}
T_{\mathrm{c}}(H) & =T_{\mathrm{c}}(0)\left[1-\left(4 \pi^{2} \xi_{0}^{2} H^{2} d^{2} / 10 \Phi_{0}^{2}\right)\right] \\
& \equiv T_{\mathrm{c}}(0)\left[1-H^{2} /\left(H_{\mathrm{c}}^{\text {grain }}\right)^{2}\right]
\end{aligned}
$$

Note that Eq. (2) implies an upturn in the field dependence of $M_{\text {dia }}$ around the magnetic field value

$$
H_{\text {up }}=\varepsilon^{1 / 2}(2.5)^{1 / 2} \Phi_{0} / \pi \xi_{0} d \equiv \varepsilon^{1 / 2} H_{\mathrm{c}}^{\text {grain }} .
$$

The solid lines in Fig. 5(a) correspond to Eq. (2), with no free parameters, using $H_{\mathrm{c}}^{\text {grain }}=1150 \mathrm{Oe}$, as extracted from the field dependence of the transition temperatures on the basis of Eq. (3). The experimental values for $H_{\text {up }}$ and for $H_{\mathrm{c}}^{\text {grain }}$ are close to those obtained from the above equations by assuming $\xi_{0}=900 \AA$, particularly if we use an effective grain size larger than the nominal one due to the size distribution. For instance, for the sample at $d=750 \AA$, Eqs. (3) and (4) yield $H_{\text {up }} \approx 1.2 \times 10^{3} \varepsilon^{1 / 2}$ Oe and $H_{\mathrm{c}}^{\text {grain }} \approx 1400$ Oe. The distribution of particle diameters is particularly detrimental for the grains of small size and introduces large errors into the estimate of the field dependence of $T_{\mathrm{c}}$ for strong magnetic fields (see Fig. 4). The zero-temperature critical field for grains where $H_{\mathrm{c}}^{\text {grain }}(T \rightarrow 0)$ is much larger than for bulk lead, since it is difficult to extrapolate the data for $T_{\mathrm{c}}(H)$ according to Eq. (3).

For sample 3 (average diameter $d=750 \AA$ ), the comparison of the field dependence of $T_{\mathrm{c}}$ (Fig. 4) with the curve derived on the basis of the temperature dependence of $H_{\mathrm{c}}{ }^{12}$ (for the sample at $d=860 \AA$ ) indicates that Eq. (2) is valid, but only for $H \leqslant H_{\mathrm{c}}^{\text {grain }} / 2$. This limit of validity could be 


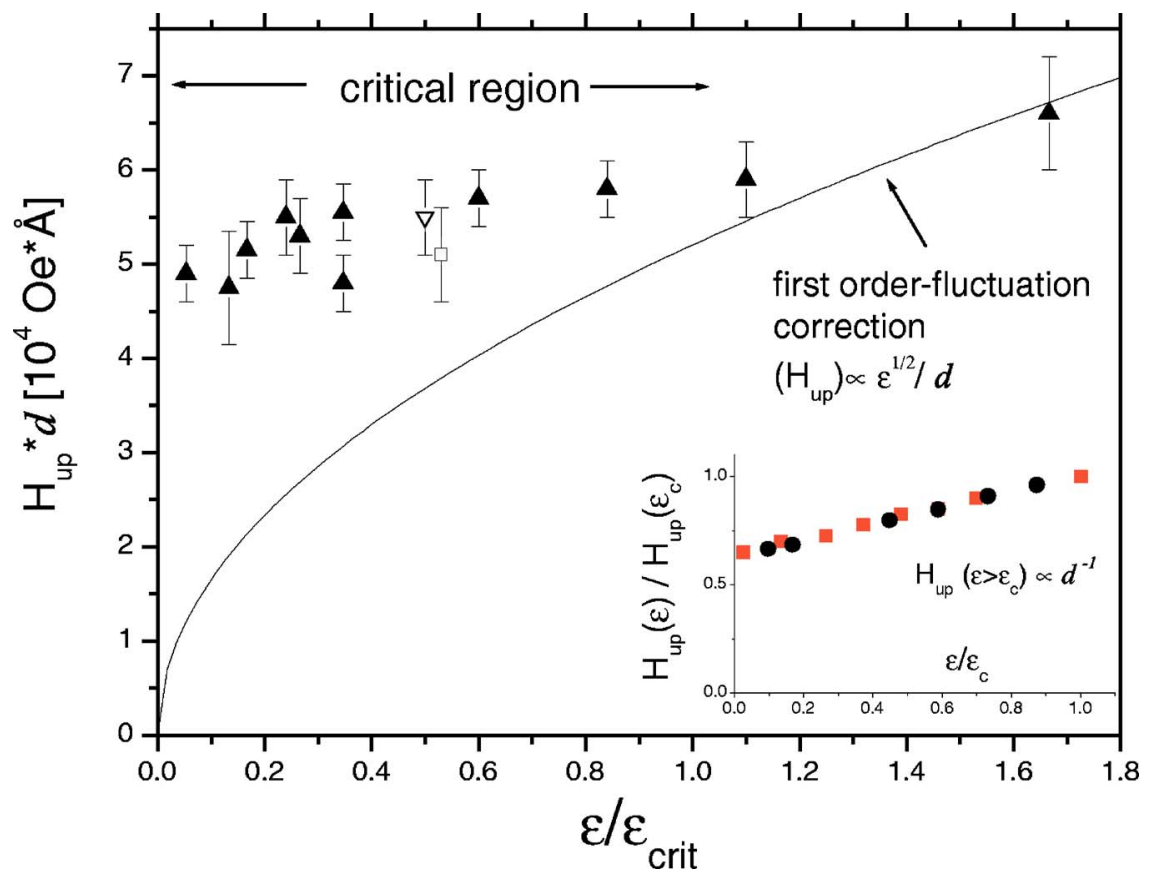

FIG. 6. (Color online) Temperature behavior of the upturn field $H_{\text {up }}$, normalized to the grain size, as a function of the reduced temperature $(\boldsymbol{\bullet}$, sample $3 ; \boldsymbol{\Delta}$, sample 1; $\square$ and $\nabla$ sample 2). The upturn field appears to scale rather well with the grain size on the whole temperature range, while the breakdown of the firstorder fluctuation correction occurs for $\varepsilon \leqslant \varepsilon_{\mathrm{c}}$. The inset shows the temperature behavior of $H_{\text {up }}$ (normalized to the value slightly above the critical temperature), as obtained from the exact expression of the GL functional and the full form of the partition function (see text).

expected since it is intrinsically related to the first-order fluctuation correction.

From Fig. 5(a) it appears that the experimental data at $T$ $=7.16 \mathrm{~K}$ are very well fitted by Eq. (2). However, for temperatures closer to $T_{\mathrm{c}}$, and particularly for $T=7.095 \mathrm{~K}$, inside the critical region, the departure of $M_{\text {dia }}$ from the behavior expected on the basis of Eq. (2) is noticeable. We shall see that the magnetization curves derived from the full form of the GL functional and of the exact partition function will account for this departure. Based on the results presented in Fig. 5(a) we believe that the isothermal magnetization curves convey information on the FD, which is much more reliable than the more commonly studied isofield data as a function of temperature (open circles).

Therefore the conclusion is that above the critical region our data are well described by the theoretical predictions for fluctuating diamagnetism as outlined above. The departures of the experimental results for $M_{\mathrm{dia}}$ with respect to the magnetization given in Eq. (2) appearing in Fig. 5(a) occur when entering the critical region, and the susceptibility is smaller than the one derived from the first-order fluctuation approximation [see Eq. (2) and the solid lines in Fig. 3].

Now we turn to the discussion of the magnetization curves in the critical region. For sample 1 the majority of the measurements were made in the temperature range where $\varepsilon$ $\leqslant \varepsilon_{\mathrm{c}}$. In the limit $H \rightarrow 0$ the magnetization is linear in the field and the susceptibility can be written

$$
\begin{aligned}
\chi_{\mathrm{dia}} & \approx-d^{3 / 2}\left(12 k_{\mathrm{B}} T_{\mathrm{c}}\right)^{1 / 2} / 33.9 \lambda_{\mathrm{L}}(0) \Phi_{0} \\
& \approx-d^{3 / 2} 0.6\left(k_{\mathrm{B}} T_{\mathrm{c}}\right)^{1 / 2} / \Phi_{0}^{2} H_{\mathrm{c}}^{\text {bulk }}
\end{aligned}
$$

which is characterized by a weak temperature dependence (see plots in Ref. 11). With the value $H_{\mathrm{c}}^{\text {bulk }}=800$ Oe for the bulk critical field, Eq. (5) yields $\chi_{\text {dia }}\left(T \approx T_{\mathrm{c}}\right)=-1.5 \times 10^{-5}$ for the sample with $d=750 \AA$, in good agreement with the data in Fig. 3. Although the observed scaling factor of $\chi_{\text {dia }}$
( $T \approx T_{\mathrm{c}}$ ) with the grain size does not follow the expected $d^{3 / 2}$ dependence, this departure is likely to be a trivial consequence of errors in our estimate for the filling factor due to the presence of the insulating oxide.

From the comparison of the solid lines with the experimental data in Fig. 5 and from the temperature dependence of the upturn field (Fig. 6), it is evident that the mean field correction breaks down below about $7.13 \mathrm{~K}$ in sample 3 and in almost the whole temperature range that has been explored in sample 1 .

In the critical region the role of the magnetic field should be discussed starting from the complete expression of the GL functional $[\mathrm{Eq}$. (1)] and by using a partition function of the form $^{1}$

$$
Z_{(0)}=\left[\pi^{3} v k_{\mathrm{B}} T / 2 b\right]^{1 / 2} \exp \left(x^{2}\right)(1-\operatorname{erf}(x)),
$$

with $x=a(H)\left(v / 2 b k_{\mathrm{B}} T\right)^{1 / 2}$, while $a(H)$, is the coefficient of the $|\psi|^{2}$ term including the factor $H^{2} d^{2} / 10$. Note that the first-order fluctuation correction derived for $\varepsilon>\varepsilon_{\mathrm{c}}$ corresponds to the approximate form of the free energy $F_{(0)}=$ $-k_{\mathrm{B}} T \ln (\pi / \alpha \varepsilon)$.

From Eq. (6) the magnetic field dependence of $M_{\text {dia }}$ can now be derived. The parameter $a(H)$ becomes $\alpha_{0} T_{\mathrm{c}}[\varepsilon$ $\left.+\left(H / H_{\mathrm{c}}^{\text {grain }}\right)^{2}\right]$, while the factor $\left(v / 2 b k_{\mathrm{B}} T\right)^{1 / 2} \alpha_{0} T_{\mathrm{c}}$ has to be estimated using the relation $\left(\alpha_{0}^{2} / b\right)=8 \pi^{2} N(0) / 7 \zeta(3)$. The resulting expression for the magnetization (per unit volume) becomes

$$
\begin{aligned}
M_{\mathrm{dia}}= & (2 \alpha / \sqrt{b} \sqrt{v}) k_{\mathrm{B}}^{3 / 2} T_{\mathrm{c}} T^{1 / 2}\left[H /\left(H_{\mathrm{c}}^{\text {grain }}\right)^{2}\right] \\
& \times\left[x-e^{x^{2}} / \sqrt{ } \pi(1-\operatorname{erf} x)\right],
\end{aligned}
$$

and $H_{\text {up }}$ can be estimated by numerical procedure. Equation (7), in the limit $H \rightarrow 0$, yields the susceptibility including the quartic term in the GL functional. 


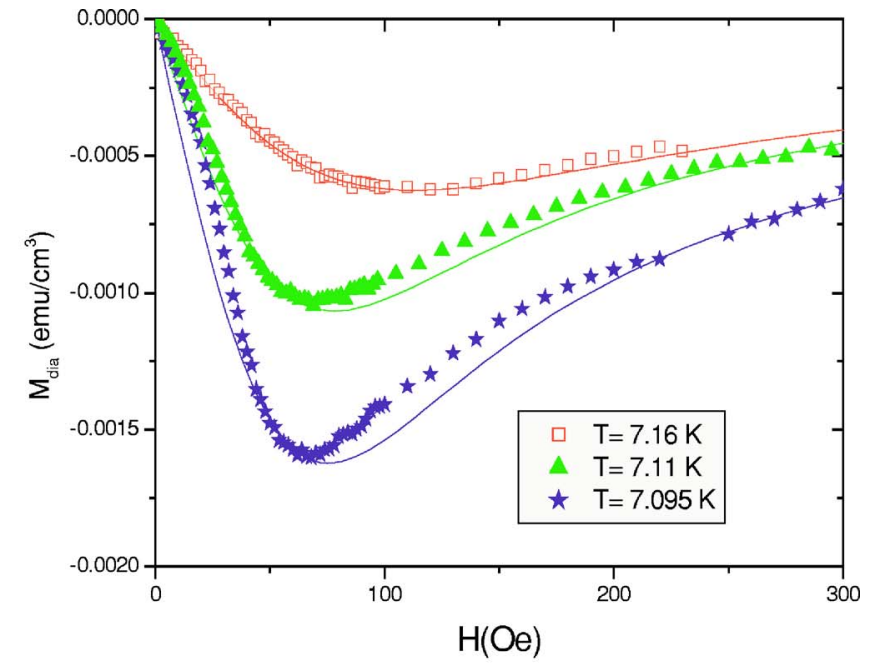

(a)

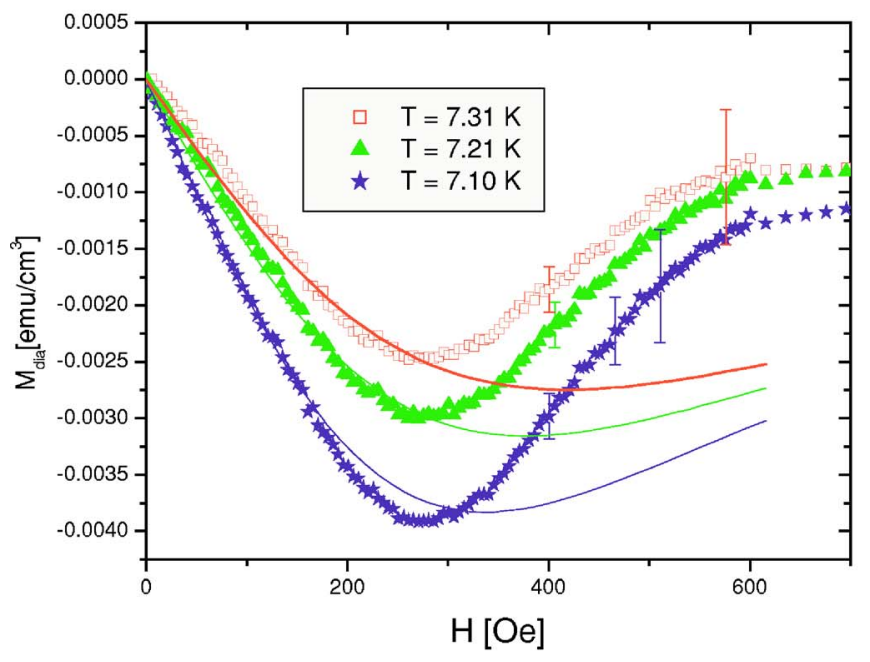

(b)

FIG. 7. (Color online) Magnetization curves at representative temperatures derived from the exact GL functional and the complete form of the zero-dimensional partition function. Part (a) of the figure refers to the sample at average diameter of the grains $750 \AA$, with the experimental data taken from Fig. 5(a). Part (b) reports the theoretical curves expected at representative temperatures for sample 1 , at average diameter size of $160 \AA$, compared with some experimental data.

In Fig. 7 the curves $M_{\text {dia }}$ vs $H$ resulting from Eq. (7) are compared with the experimental data for several temperatures. Note that for sample 3 at $T=7.16 \mathrm{~K}$ the theoretical curve coincides with the one shown in Fig. 5(a), as expected since at this temperature $\varepsilon>\varepsilon_{\mathrm{c}}$. However, for the other two temperatures significant differences appear with respect to the theoretical calculations shown in Fig. 5(a). Using the complete form of the partition function, the resulting magnetization curves are in very good agreement with the experimental findings, particularly for $M_{\text {dia }}\left(H \approx H_{\text {up }}\right)$, having kept all the parameters unchanged. Thus, the role of the term $|\Psi|^{4}$ in the GL functional is crucial in the critical region and must be included to quantitatively describe the data.
The theoretical curves derived for the sample at $d$ $=160 \AA$ are reported in Fig. 7(b). From comparison with the magnetization data one can observe that the trend of $M_{\text {dia }}$ vs $H$ is rather well reproduced up to a field strength of the order of $H_{\text {up }}$ where we have used the value $H_{\mathrm{c}}^{\text {grain }}=2500$ Oe for the critical field (Table I). For small grain size the theoretical curves are very sensitive to the value of $H_{\mathrm{c}}^{\text {grain }}$ through the coefficient $a(H)$ in Eq. (6) which is difficult to determine precisely by means of Eq. (3) on the basis of the field dependence of $T_{\mathrm{c}}$. Nonetheless, the value of the upturn field and the magnetization for $H=H_{\text {up }}$ is rather well accounted for by our calculations. Upon increasing the field above $H_{\text {up }}$ the experimental data appear to decrease toward zero faster than the theoretical predictions. An attempt to take into account the size distribution of the grains reported in Fig. 1(d) does not lead to any significant improvement of the theoretically predicted behavior at strong fields. On the other hand, one should note that at strong fields the experimental error increases rapidly as a consequence of the subtraction procedure explained in the Experimental section. Furthermore the smallest grains in the distribution of the diameters quantum size effects could drastically modify the superconducting properties, ${ }^{16,1}$ which in turn could produce the discrepancy between theory and experiment for $H>H_{\text {up }}$. Attempts are now underway to obtain powders with a narrower distribution in diameters of the grains.

From the behavior of $M_{\text {dia }}$ in the critical region as shown in Fig. 7(b) one can see that the upturn field increases with increasing temperature while the diamagnetic magnetization $M_{\text {dia }}\left(H=H_{\text {up }}\right)$ increases when $H_{\text {up }}$ decreases. This implies that the qualitative behavior predicted for the magnetization curves by the first-order correction theory is still valid even in the critical region, although the temperature dependence of the susceptibility and of the upturn field are strongly modified. This is reflected in Fig. 6, since by scaling the reduced temperature in terms of $\varepsilon_{\mathrm{c}}$ for each size, the quantity $\left(H_{\mathrm{up}} d\right)$ approximately keeps a size-independent value, in spite of the breakdown of the mean field result $H_{\text {up }} \propto \varepsilon^{1 / 2} / d$. The inset in Fig. 6 shows the temperature dependence of $H_{\text {up }}$ (normalized to the value slightly above $\varepsilon_{\mathrm{c}}$ ) expected in the critical region according to our derivation based on the exact GL functional and the full form of the partition function. The experimental findings for $\left(H_{\mathrm{up}} d\right)$ vs $\varepsilon / \varepsilon_{\mathrm{c}}$ are well described by our calculations.

\section{SUMMARIZING REMARKS}

By means of magnetization measurements we have studied the superconducting fluctuations and the related fluctuation diamagnetism above the superconducting transition temperature in $\mathrm{Pb}$ nanoparticles of size smaller than the coherence length. The isothermal field dependence of the diamagnetic magnetization $M_{\mathrm{dia}}$ above $T_{\mathrm{c}}$ has been discussed in the framework of exact theories based on the GinzburgLandau functional in the zero-dimensional condition.

The first-order fluctuation correction is found to be valid only outside the critical region $\varepsilon>\varepsilon_{\mathrm{c}}$, where it accurately describes the behavior $M_{\text {dia }}$ for magnetic fields $H$ not too close to the critical field. Also, the scaling properties of 
$d T_{\mathrm{c}}(H) / d H$ for small fields and of the upturn field $H_{\text {up }}$ in the magnetization curves are well described within that approximation.

In the critical region, however, the role of the field and the limits of validity of the first-order fluctuation correction have been analyzed by comparing the experimental findings to the derivation of $M_{\mathrm{dia}}$ as a function of the magnetic field starting from the complete form of the GL functional and with the exact expression of the zero-dimensional partition function. We find that the role of the $|\Psi|^{4}$ term in the GL functional is crucial in describing the data in the critical region. For the sample with average grain diameter of $750 \AA$, the fluctuating diamagnetism can be well described by our extended model even in the critical region, without introducing any adjustable parameters. For the sample with the smallest average diameter of $160 \AA$, the agreement of the numerically derived $M_{\text {dia }}$ with the experimental findings is again good for fields of the order of $H_{\text {up }}$. Poor agreement between the theoretically predicted $M_{\text {dia }}$ vs $H$ and our data is observed for fields above $H_{\text {up }}$, when the fluctuating diamagnetic contribution is approaching zero and the subtraction procedure of the paramagnetic term introduces large errors.

The temperature dependence of the upturn field and the scaling properties with the grain size are also well described by our calculations both outside and inside the critical region, with the product $\left(H_{\mathrm{up}} d\right)$ vs reduced temperature being approximately size-independent and following the predicted temperature dependence, even though the mean field result $H_{\text {up }} \propto\left(\varepsilon^{1 / 2} / d\right)$ evidently breaks down. The relevance of the magnetization curves $M_{\text {dia }}$ vs $H$ and of the upturn field $H_{\text {up }}$ for the study of the fluctuating diamagnetism above the superconducting transition temperature has been emphasized.

\section{ACKNOWLEDGMENTS}

Preliminary measurements carried out by I. Zucca are acknowledged. W.-H. Li, National Central University of Chung- $\mathrm{Li}$, is thanked for the loan of a batch of $\mathrm{Pb}$ nanoparticles, particularly sample (2b) (see Table I), used for comparison. A. A. Varlamov is gratefully thanked for stimulating discussions and for having introduced the authors to the study of the superconducting fluctuations. The authors also thank the Centre CISME (Centro Interdipartimentale di Servizio per la Microscopia Elettronica) of the "Federico II" University for access to their TEM. Useful discussions with P. Carretta are acknowledged. Mike Graf (Boston College) is thanked for critical reading of the manuscript. The work has been carried out in the framework of a FIRB-MIUR project "microsistemi basati su materiali magnetici innovativi strutturati su scala nanoscopica” (Pnr 2001-2003).
*Present address: Institute of General Physiology and Biological Chemistry, University of Milano, Via Trentacoste 2, I-20134 Milano, Italy.

${ }^{1}$ See A. I. Larkin and A. A. Varlamov, in The Physics of Superconductors, edited by K. H. Benneman and J. B. Ketterson (Springer-Verlag, Berlin, 2003), Vol. 1, pp. 95-231; The Theory Fluctuations in Superconductors (Oxford, United Kingdom, 2005).

${ }^{2}$ P. Mosconi, A. Rigamonti, and A. A. Varlamov, Appl. Magn. Reson. 19, 345 (2000).

${ }^{3}$ M. Tinkham, Introduction to Superconductivity (McGraw-Hill, New York, 1996), Chap. 8, and references therein.

${ }^{4}$ A. Lascialfari, T. Mishonov, A. Rigamonti, P. Tedesco, and A. A. Varlamov, Phys. Rev. B 65, 180501 (2002).

${ }^{5}$ A. Lascialfari, A. Rigamonti, L. Romanó, P. Tedesco, A. A. Varlamov, and D. Embriaco, Phys. Rev. B 65, 144523 (2002).

${ }^{6}$ For an exhaustive review on the NMR studies in BCS superconductors, see D. E. MacLaughlin, Solid State Phys. 31, 63 (1976).

${ }^{7}$ For a mise a point of the theory and a critical review of the experiments, see E. Simanek, in Local Properties at Phase Transitions, edited by K. A. Muller and A. Rigamonti (NorthHolland, Amsterdam, 1976), pp. 838-855.
${ }^{8}$ R. A. Buhrman and W. P. Halperin, Phys. Rev. Lett. 30, 692 (1973).

${ }^{9}$ S. Kobayashi, T. Takahashi, and W. Sasaki, J. Phys. Soc. Jpn. 36, 714 (1974).

${ }^{10} \mathrm{~V}$. V. Shmidt, in Proceedings of the IXX International Conference on Low Temperature Physics, edited by M. P. Malkov, L. P. Pitaesvski, and A. Shalnikov (Viniti Publishing House, Moscow, 1967), Vol. II B, p. 205.

${ }^{11}$ B. Muhlschegel, D. J. Scalapino, and R. Denton, Phys. Rev. B 6, 1767 (1972).

${ }^{12}$ W-H. Li, C. C. Yang, F. C. Tsao, and K. S. Lee, Phys. Rev. B 68 , 184507 (2003).

${ }^{13}$ C. T. Black, D. C. Ralph, and M. Tinkham, Phys. Rev. Lett. 76, 688 (1996)

${ }^{14}$ A. P. Tsai, N. Chandrasekhar, and K. Chattopadhyay, Appl. Phys. Lett. 75, 1527 (1999).

${ }^{15}$ V. N. Gladilin, V. M. Fomin, and J. T. Devruse, Phys. Rev. B 70, 144506 (2004).

${ }^{16}$ J. von Delft, Ann. Phys. (Leipzig) 10, 1 (2001); J. von Delft and D. C. Ralph, Phys. Rep. 345, 61 (2001).

${ }^{17}$ T. Mishonov and E. Penev, Int. J. Mod. Phys. B 14, 3831 (2000).

${ }^{18}$ Figlarz et al., U.S. Patent No. 4,539,041 (3 September 1985). 